Boise State University

ScholarWorks

Geosciences Faculty Publications and

Presentations

Department of Geosciences

$9-2016$

Isolating Retrograde and Prograde Rayleigh-Wave Modes Using a Polarity Mute

Gabriel Gribler

Boise State University

Lee M. Liberty

Boise State University

T. Dylan Mikesell

Boise State University

Paul Michaels

Boise State University

This document was originally published in Geophysics by the Society of Exploration Geophysicists. Copyright restrictions may apply. doi: 10.1190/geo2015-0683.1 


\title{
Isolating retrograde and prograde Rayleigh-wave modes using a polarity mute
}

\author{
Gabriel Gribler $^{1}$, Lee M. Liberty ${ }^{1}$, T. Dylan Mikesell ${ }^{1}$, and Paul Michaels ${ }^{1}$
}

\begin{abstract}
Estimates of S-wave velocity with depth from Rayleighwave dispersion data are limited by the accuracy of fundamental and/or higher mode signal identification. In many scenarios, the fundamental mode propagates in retrograde motion, whereas higher modes propagate in prograde motion. This difference in particle motion (or polarity) can be used by joint analysis of vertical and horizontal inline recordings. We have developed a novel method that isolates modes by separating prograde and retrograde motions; we call this a polarity mute. Applying this polarity mute prior to traditional multichannel analysis of surface wave (MASW) analysis improves phase velocity estimation for fundamental and higher mode dispersion. This approach, in turn, should lead to improvement of S-wave velocity estimates with depth. With two simple models and a field example, we have highlighted the complexity of the Rayleigh-wave particle motions and determined improved MASW dispersion images using the polarity mute. Our results show that we can separate prograde and retrograde signals to independently process fundamental and higher mode signals, in turn allowing us to identify lower frequency dispersion when compared with single component data. These examples demonstrate that the polarity mute approach can improve estimates of S-wave velocities with depth.
\end{abstract}

\section{INTRODUCTION}

The Rayleigh waves are surface seismic waves that propagate with an elliptical particle motion due to the mix of $\mathrm{P}$ - and $\mathrm{S}_{\mathrm{V}}$-wave components (Rayleigh, 1885). The Rayleigh waves become dispersive in layered media due to longer wavelengths (lower frequencies) propagating at greater depths, causing the well-known dispersion relationship between frequency and phase velocity (Dorman and Ewing, 1962; Aki and Richards, 1980). The dispersion of the Rayleigh waves is the basis for the methods of spectral analysis of surface waves (Stokoe and Nazarian, 1983) and multichannel analysis of surface waves (Park et al., 1999), which use active-source vertical component particle velocity fields to estimate phase velocity as a function of frequency. Rayleigh waves traveling at a given velocity can propagate at several different frequencies, or modes, similar to a fixed oscillating string. The lowest frequency oscillation is defined as the fundamental mode, whereas all of the higher frequencies are known as higher modes (Garland, 1979; Aki and Richards, 1980; Xia et al., 2003).

Although the fundamental mode alone can be used to estimate S-wave velocity at depth, higher modes can provide additional constraints to improve the estimate of complex subsurface velocity distributions (Casto et al., 2009, 2010). Under many geologic conditions, fundamental and higher modes may be difficult to identify, isolate, and measure (Ivanov et al., 2011; Dal Moro et al., 2015), and work has been done to better isolate the fundamental and higher Rayleigh-wave modes (Luo et al., 2009). In the case of higher modes interfering with the fundamental mode, Zhang (2011) presents a method that models the effective dispersion curve measured due to interference. In the same vain as previous researchers, we present a new method to improve the measurement of individual mode velocity dispersion for the fundamental and higher mode Rayleigh waves by isolating wavefields based on particle motions. Our approach leads to higher confidence dispersion curve estimation compared with single component analyses and can improve S-wave velocity estimates with depth.

Fundamental mode displacements at the free surface propagate almost exclusively in retrograde motion (Aki and Richards, 1980); however, for a few rare cases, there is a potential for prograde motion at frequencies ranging from 0.1 to $0.3 \mathrm{~Hz}$ in earth models (Mooney and Bolt, 1966; Tanimoto and Rivera, 2005; Malischewsky et al., 2008). The higher mode Rayleigh waves propagate in either retrograde or prograde motions (Jones et al., 1963); however, it has been

Manuscript received by the Editor 26 December 2015; revised manuscript received 2 May 2016; published online 11 August 2016.

${ }^{1}$ Boise State University, Department of Geosciences, Boise, Idaho, USA. E-mail: gabegribler@boisestate.edu; lliberty@boisestate.edu; dylanmikesell@ boisestate.edu; paulmichaels@boisestate.edu.

(C) 2016 Society of Exploration Geophysicists. All rights reserved. 
found that higher modes commonly propagate in prograde motion below $50 \mathrm{~Hz}$ (De Nil, 2005). In our study, we use a 2D receiver array and model 2C (vertical and horizontal inline) shot records for 1D velocity models (Figure 1).

In these simple models, higher modes propagate in prograde motion, whereas the fundamental mode propagates in retrograde motion. With $2 \mathrm{C}$ recordings, we convert the motions to polar coordinates and determine the dominate particle motion through time at each station. The polarity mute we then apply simply mutes sections of the time series in which retrograde or prograde motion dominates, thus isolating the waves propagating in those polarities. Our first model (model 1) has a vertical velocity gradient that is typical of many unconsolidated sedimentary basins. Our second model (model 2) has a large velocity contrast at $10 \mathrm{~m}$ depth, separating two-constant velocity layers, which is typical of a shallow bedrock environment. We then present a velocity gradient field example from the Long Valley, Idaho (Liberty and Gribler, 2014), sedimentary basin collected with a land-streamer system to highlight the benefit of multicomponent surface wave data collection and analysis. These examples demonstrate that we can isolate and separate the fundamental mode from higher modes in cases when they have different particle motions using the multicomponent polarity mute.

\section{POLARITY MUTE THEORY}

To investigate the elliptical Rayleigh-wave particle motions, we generate synthetic shot gathers with a $1-100 \mathrm{~Hz}$ frequency range. The algorithm used to produce these synthetic shot gathers is that of Michaels and Smith (1997), which is derived from Aki and Richards (1980). This code models individual Rayleigh-wave modes and enables a direct comparison between particle motions of fundamental and higher modes. To illustrate our method, we use model 1 (Figure 1a) and generate 2C shot gathers that contain only the fundamental and first higher mode (Figure $2 \mathrm{a}$ and $2 \mathrm{~b}$ ). In model 2 (Figure 1b), we include the fundamental and first nine higher modes (Figure $2 \mathrm{~d}$ and $2 \mathrm{e}$ ). In both synthetic shot gathers, the receiver spacing is $1 \mathrm{~m}$ (99 receivers in total) with $1 \mathrm{~m}$ between the shot and first receiver.

To identify the different polarities of the Rayleigh-wave particle motion (i.e., retrograde or prograde), we convert the vertical $V$ and horizontal inline $H$ time series from Cartesian coordinates into polar coordinates with

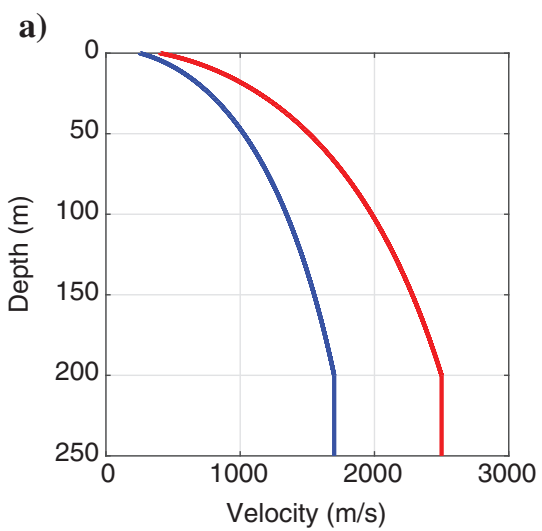

b)

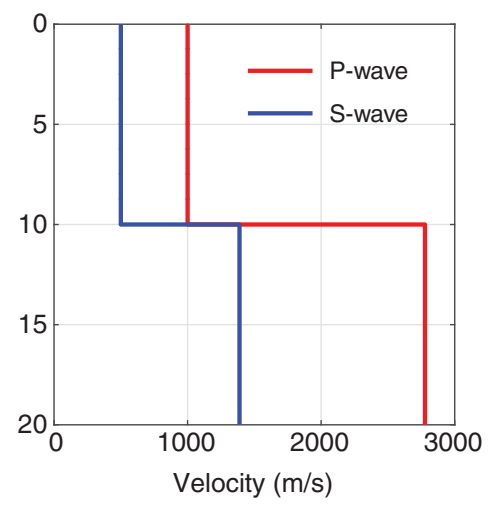

Figure 1. (a) Model 1: gradient velocity model and (b) model 2: shallow bedrock velocity model.

$$
\begin{gathered}
A(t)=\sqrt{V(t)^{2}+H(t)^{2}}, \\
\Phi(t)=\tan ^{-1}\left(\frac{V(t)}{H(t)}\right),
\end{gathered}
$$

where amplitude $A$ and phase angle $\Phi$ are the polar coordinates. Our sign convention is defined as positive vertical motion $V$ downward and positive horizontal motion $H$ outward from the source location in the inline direction. With this sign convention, the Rayleigh waves propagating with retrograde motion have a decreasing unwrapped phase angle with an increasing time. Conversely, prograde motion represents an increasing unwrapped phase angle with increasing time. We determine the instantaneous Rayleigh-wave particle motion at a given offset from the phase angle at a given time sample after unwrapping the phase angle time series. Retrograde motion will yield a negative slope and prograde motion will yield a positive slope in this times series. Before extracting the dominant trend, we apply a five sample long 1D convolutional smoothing filter to the unwrapped phase angle data to suppress high-frequency phase fluctuations (Figure $2 \mathrm{c}$ and $2 \mathrm{f}$ ).

From model 1, we confirm retrograde fundamental mode surface wave particle motion if we consider the instantaneous particle motion (Figure 3a) over all traces. When we generate a shot gather with only the first higher mode Rayleigh wave, we observe predominately prograde motion (Figure 3b), and when the fundamental and first higher modes are combined, regions of predominately retrograde and prograde signals are separated by apparent velocity (Figure $3 \mathrm{c}$ ).

We isolate retrograde and prograde arrivals by muting sections of the waveforms based on this unwrapped phase slope. To remove prograde motion, we mute data samples in the $V$ and $H$ recordings that have a positive unwrapped phase slope by setting those samples to zero. Conversely, to remove retrograde motions, we mute $V$ and $H$ data samples with a negative unwrapped phase slope. At this point, we must point out an apparent limitation to this method. When we apply the polar mute to remove the prograde (higher mode) signal, some of the low-frequency component of the fundamental mode may be lost due to overlap between low-frequency retrograde and prograde motions. This is a limitation of applying this technique in the time domain. Conversely, when the retrograde signal is removed, the low-frequency component of the first higher mode may be lost. This model-dependent data loss becomes apparent in the following dispersion images and will be discussed further in the remaining sections.

\section{VELOCITY-GRADIENT MODEL}

To understand the influence of the polar mute in phase velocity estimation, we transform the model 1 shot gathers (Figure $2 \mathrm{a}$ and $2 \mathrm{~b}$ ) into phase velocity-frequency coherence plots (i.e., dispersion images) using the phase-shift transform method (Park et al., 1999). We compare the dispersion images (Figure 4) with the calculated theoretical dispersion curves for fundamental and first higher modes. The dispersion images from the $V$ and $H$ component data show high coherence along only 
the predicted fundamental mode (black curve, Figure $4 a$ and $4 b)$. The higher mode dispersion is difficult to identify because of low-signal coherence and fundamental mode side lobe artifacts introduced by the velocity-frequency transform.

We apply the same phase velocity-frequency transform to the vertical and horizontal components after applying the prograde polarity mute to isolate the retrograde signal (Figure $4 \mathrm{c}$ and $4 d$ ). First, we note that the higher mode signal has been suppressed. Second, we note that both of these dispersion images match the calculated fundamental mode dispersion but only greater than $10 \mathrm{~Hz}$. This is a direct consequence of the overlapping low-frequency components mentioned in the previous section. The lost low-frequency retro-
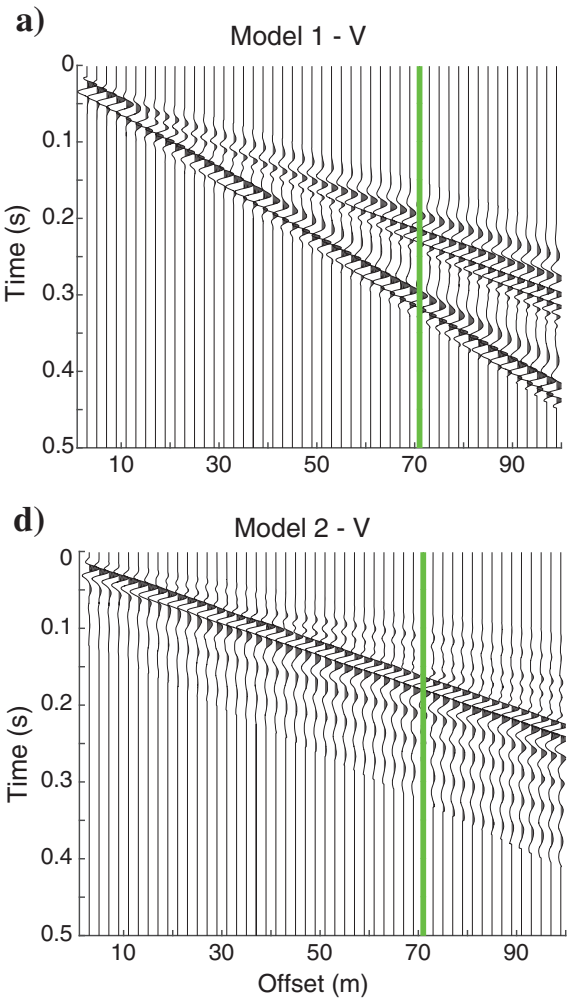

b)

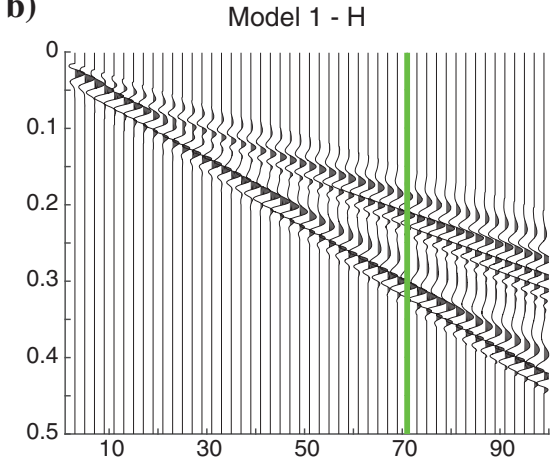

e)

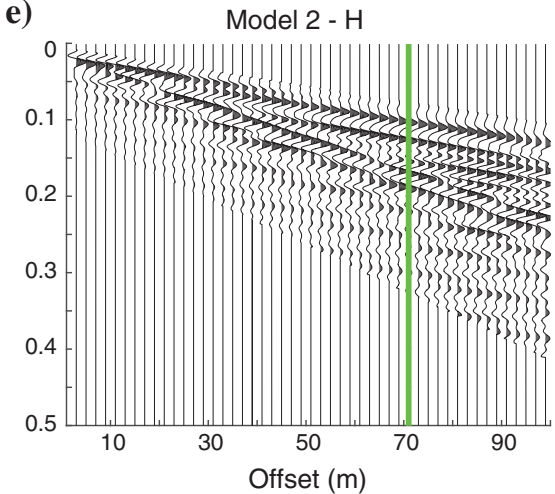

c)

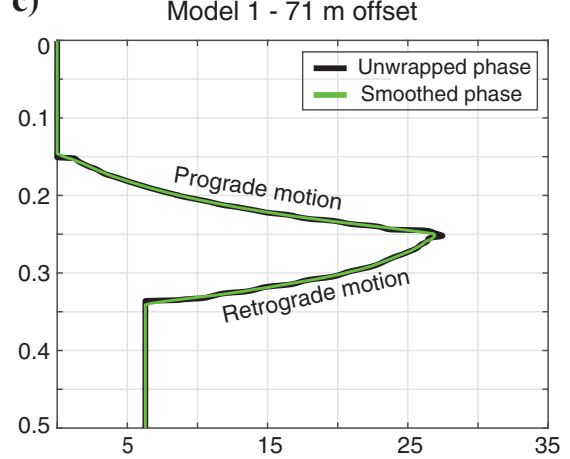

f)

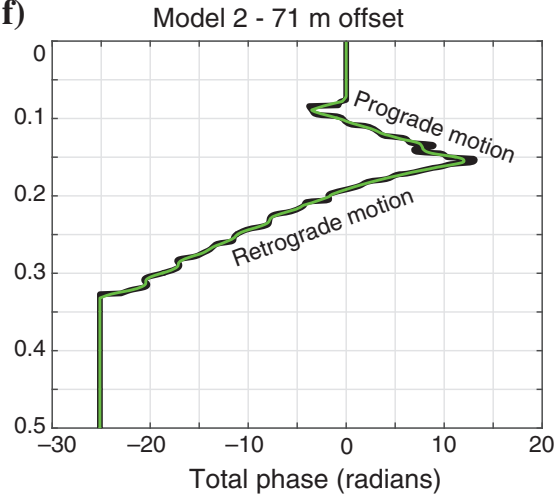

Figure 2. (a) Vertical component Rayleigh-wave synthetic shot gather for model 1 (gradient). The green line denotes trace highlighted in (c). (b) Horizontal component shot gather for model 1. (c) Unwrapped phase from the $71 \mathrm{~m}$ offset trace. The black line denotes unwrapped phase and green line denotes the smoothed version (see text). Positive slope indicates prograde motion and negative slope indicates retrograde motion. (d) Vertical component shot gather for model 2 (two-layer model). (e) Horizontal component shot gather for model 2. (f) Unwrapped phase from the $71 \mathrm{~m}$ offset trace. An automatic gain control with a $0.15 \mathrm{~s}$ window is applied in (d and e) for display purposes only.

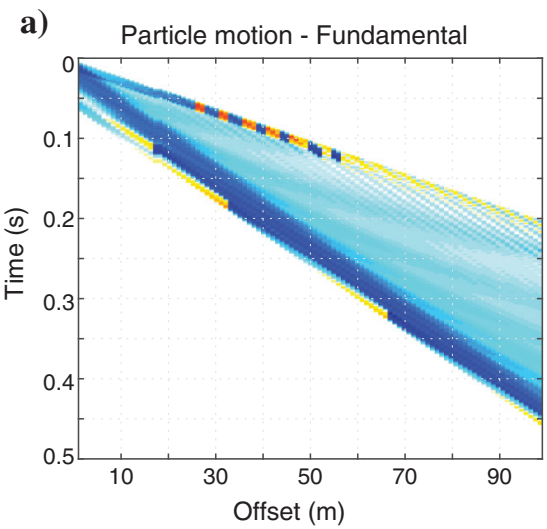

b) Particle motion - First higher mode

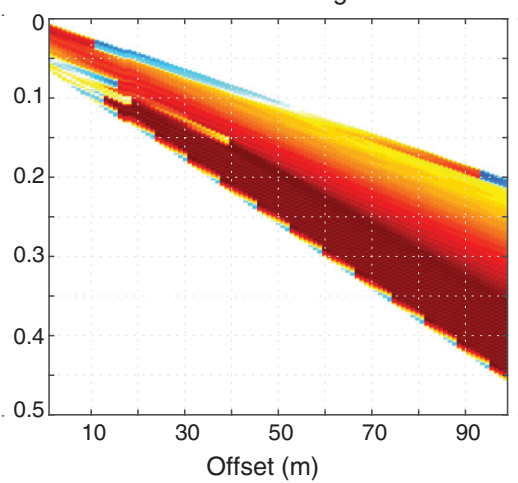

c) Particle motion - Combined

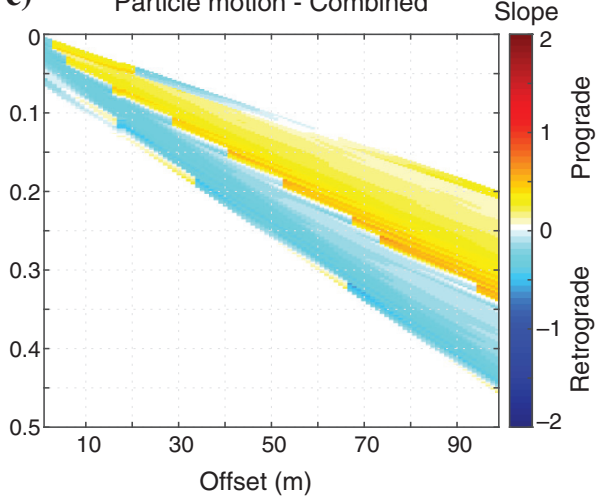

Figure 3. (a) Instantaneous particle motion for synthetic shot gathers from model 1 that contains only the fundamental mode. (b) Same as (a) but for the first higher mode only. (c) Instantaneous particle motion for the shot gather containing fundamental and first higher mode (Figure $2 \mathrm{a}$ and $2 \mathrm{~b}$ ). The particle motion is determined by the slope of the unwrapped phase (Figure 2c) across all offsets. Blue denotes retrograde motion and red denotes prograde motion. In this model, the fundamental mode propagates in retrograde motion, whereas the first higher mode propagates predominantly in prograde motion. 
grade energy can be seen in the prograde signal dispersion images (Figure $4 \mathrm{e}$ and $4 \mathrm{f}$ ). These images present the dispersion images from shot gathers after muting the retrograde signals. The higher mode dispersion is now the highest coherency signal greater than $15 \mathrm{~Hz}$ and tracks the theoretical first higher mode dispersion (red) curve. The leaked fundamental mode energy appears below $15 \mathrm{~Hz}$. In the frequency bands in which the fundamental and higher mode energies do not overlap, it is quite clear that the polarity mute has improved higher mode dispersion coherence and allows one now to readily pick the first higher mode dispersion curve.

\section{TWO-LAYER MODEL}

Dal Moro et al. (2015) demonstrate the misidentification of the Rayleigh-wave modes in a high-velocity contrast (shallow bedrock) model, in which fundamental mode dispersion shows low-relative phase velocity-frequency coherence compared with the first higher mode. Also noted in this study is the potential for convergence (or interference) of the fundamental and higher modes. The dispersion images for the model $2 \mathrm{~V}$ and $H$ data demonstrate this observation (Figure 5a and 5b). Although the highest coherence in the vertical component dispersion image follows the theoretical fundamental mode dispersion curve at high frequencies, the fundamental and first higher modes begin to interfere below $20 \mathrm{~Hz}$. The horizontal component dispersion image displays increased coherence of the first higher mode compared with the fundamental mode signal at high frequencies and also displays increased coherence of the other higher modes. In a similar model, Dal Moro et al. (2015) observe greater coherence in the higher modes when compared with the fundamental mode with vertical component data. This again highlights the need for multicomponent data to help identify the different modes in this geologic setting. Furthermore, Casto et al. (2009) suggest that velocity estimates at a site with a thin low-velocity layer over a high-velocity layer would be improved by incorporating the higher mode dispersion information into the S-wave velocity analysis.

Following the example in model 1, we apply the prograde and retrograde polarity mutes to the $V$ and $H$ data prior to computing dispersion images. After applying the prograde polarity mute to isolate the retrograde signal (Figure $5 \mathrm{c}$ and $5 \mathrm{~d}$ ), we see that higher mode signals are suppressed and the fundamental mode is more coherent greater than $20 \mathrm{~Hz}$. After applying the retrograde mute to isolate the prograde signal, the first higher mode has greater coherence (Figure $5 \mathrm{e}$ and 5f). This improved coherency is best observed on the horizontal component dispersion plot in which high coherence signal matches the theoretical dispersion plot down to $20 \mathrm{~Hz}$ in which interference with the fundamental mode begins. The other higher modes also show improved coherence; however, we do not explore these further. This velocity model highlights the benefit of the polarity mute as improved phase velocity-frequency coherence is observed on vertical and horizontal dispersion images and modes are isolated.

\section{FIELD DATA EXAMPLE}

We present field data collected along a gravel road in Donnelly, Idaho, as part of an earthquake site-response study (Liberty and Gribler, 2014). Liberty and Gribler (2014) estimate S-wave velocities
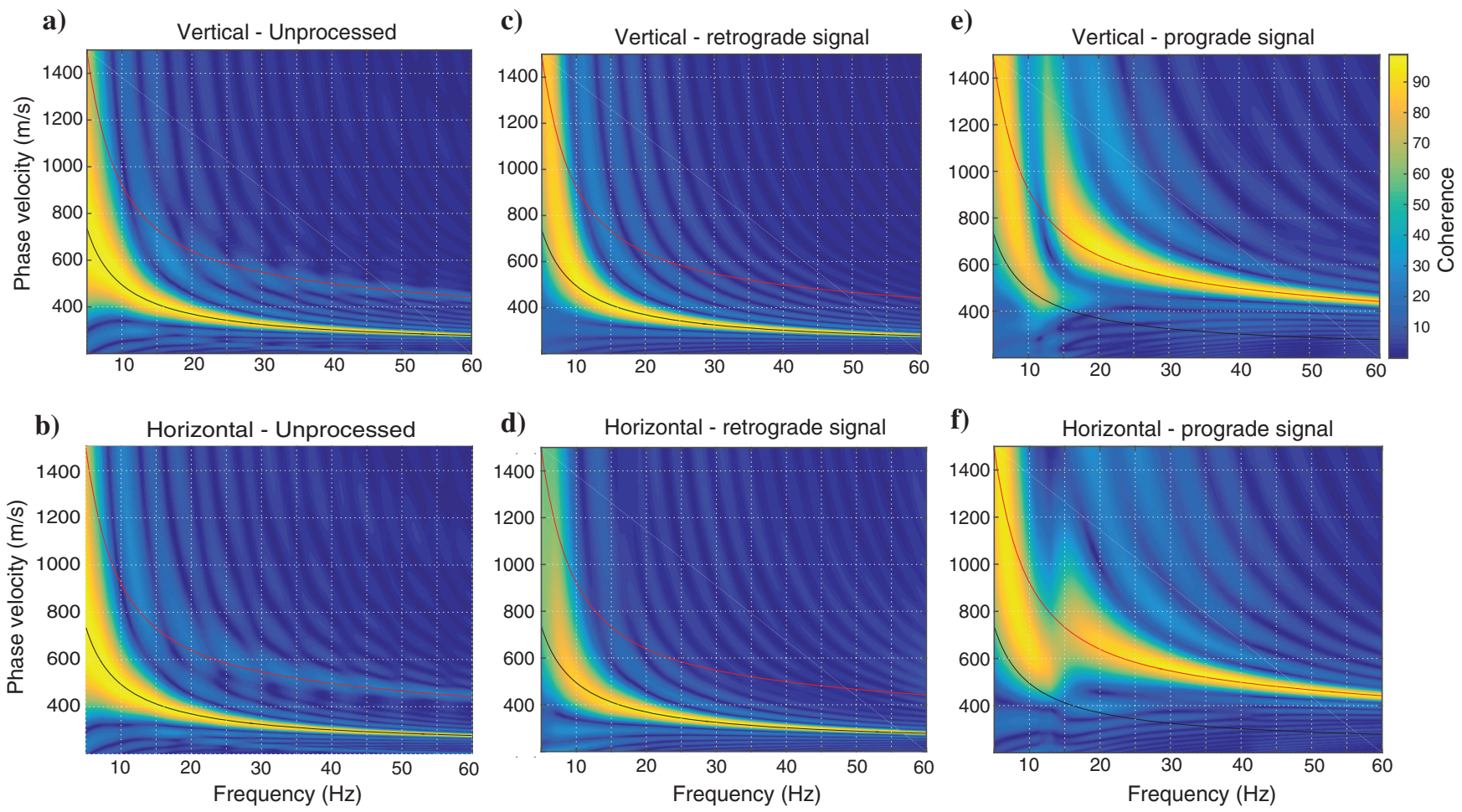

Figure 4. Phase velocity versus frequency coherence plots for model 1 (Figure 1a) with yellow denoting high coherence and dark blue denoting low coherence. The black line denotes the retrograde propagating fundamental mode dispersion curve and red line denotes the prograde propagating first higher mode. (a) Unprocessed vertical component data, (b) unprocessed horizontal component, (c) vertical component after prograde polarity mute, (d) horizontal component after prograde polarity mute, (e) vertical component after retrograde polarity mute, and (f) horizontal component after retrograde polarity mute. Color scale indicates the relative coherence in each plot. 
that increase from approximately $160 \mathrm{~m} / \mathrm{s}$ at the surface to approximately $650 \mathrm{~m} / \mathrm{s}$ at $40 \mathrm{~m}$ depth at this site. This velocity gradient of $12.25 \mathrm{~m} / \mathrm{s} / \mathrm{m}$ is consistent with soft soil grading to soft rock. Multicomponent seismic data were acquired using a 48 station land streamer with $1 \mathrm{~m}$ station spacing. Each station was composed of $4.5 \mathrm{~Hz}$ vertical and horizontal inline component geophones coupled to the ground with flat base plates. The source was a $500 \mathrm{lb}$ accelerated weight drop and a $4 \mathrm{~m}$ source to first receiver offset.

A top mute is applied to the raw shot gathers to suppress body waves arriving before the surface waves (red line, Figure 6). Following the two previous sections, we compute the dispersion images of raw, retrograde, and prograde signals (Figure 7). Above approximately $15 \mathrm{~Hz}$, the raw vertical and horizontal component dispersion images give fundamental mode phase velocities between 175 and 200 m/s (Figure 7a and 7b). At lower frequencies, the vertical and horizontal components have different dispersion trends. Moreover, the vertical component has lower relative coherence along the presumed fundamental mode when compared with that of the horizontal component.

Between 7 and $15 \mathrm{~Hz}$, the vertical component coherency indicates little change in phase velocity of the fundamental mode (Figure 7a). This is a surprising observation and likely indicates interference by a body wave or a higher mode. In this same frequency range, the horizontal component indicates a more typical dispersive trend with an increase in phase velocity as frequency decreases. Interestingly, the horizontal component dispersion image shows a sharp increase in phase velocity at $10 \mathrm{~Hz}$ and then flattens between 5 and $7 \mathrm{~Hz}$ (Figure 7b). This also does not follow the anticipated dispersion of the fundamental mode within a sedimentary basin and indicates that there may be interference from other modes. Higher mode signals have low relative coherence, which are difficult to identify on either vertical or horizontal component dispersion images.

If we isolate the retrograde signals via the polarity mute, we isolate the fundamental mode to approximately $7 \mathrm{~Hz}$ on the vertical component (Figure 7c), whereas the horizontal component (Figure 7d) shows a coherent dispersion trend down to $5 \mathrm{~Hz}$. There is also a reduced coherency in the higher mode signals after muting the data. The substantial improvement in lower frequency coherency allows for a more confident pick in phase velocity down to approximately $5 \mathrm{~Hz}$ rather than $10 \mathrm{~Hz}$ and would lead to improved $\mathrm{S}$-wave velocity estimates at greater depths. The reason for this observed improvement in the dispersion coherence is due to isolation of the retrograde fundamental mode by the prograde polarity mute.

To isolate the prograde higher modes, we apply the retrograde polarity mute to the vertical and horizontal component data. The resulting dispersion images (Figure 7e and 7f) show improved higher mode dispersive trends, with the horizontal component showing more coherent signals at lower frequencies. When the prograde modes are isolated from the retrograde modes, we observe that low-frequency prograde signals approach the fundamental mode velocities. This convergence indicates that the fundamental mode and higher modes are interfering just as in our model 2 example. Using the multicomponent polarity mute allows us to isolate the various modes
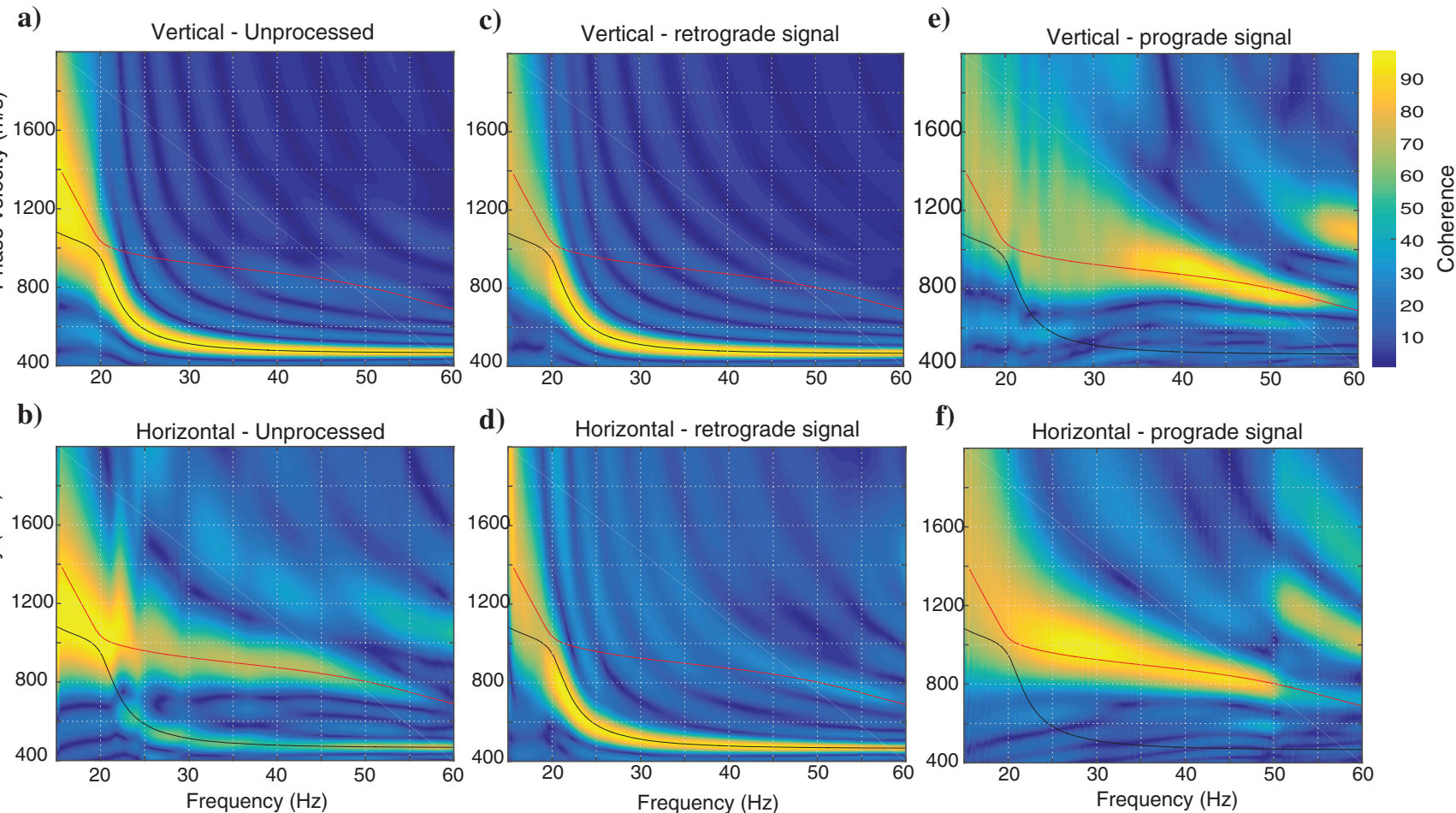

Figure 5. Phase velocity versus frequency coherence images for the shallow bedrock velocity model (Figure 1b) with yellow denoting high coherence and dark blue denoting low coherence. The black line denotes the retrograde propagating fundamental mode and red line denotes the prograde propagating first higher mode. (a) Unprocessed vertical component data, (b) unprocessed horizontal component, (c) vertical component after prograde polarity mute, (d) horizontal component after prograde polarity mute, (e) vertical component after retrograde polarity mute, and (f) horizontal component after retrograde polarity mute. Color scale indicates the coherence relative to maximum coherence in each plot. 
and estimate phase velocity dispersion for higher modes; something that would not have been possible using single-component data.

\section{DISCUSSION}

This new type of analysis is a compelling justification for multicomponent recordings of surface waves. The field data example demonstrates that the vertical component dispersion image alone fails to accurately capture the fundamental mode Rayleigh-wave dispersion. In this example, and in the two-layer example, the horizontal inline component gives better dispersion constraints when compared with the vertical component. However, using a $2 \mathrm{C}$ polar-
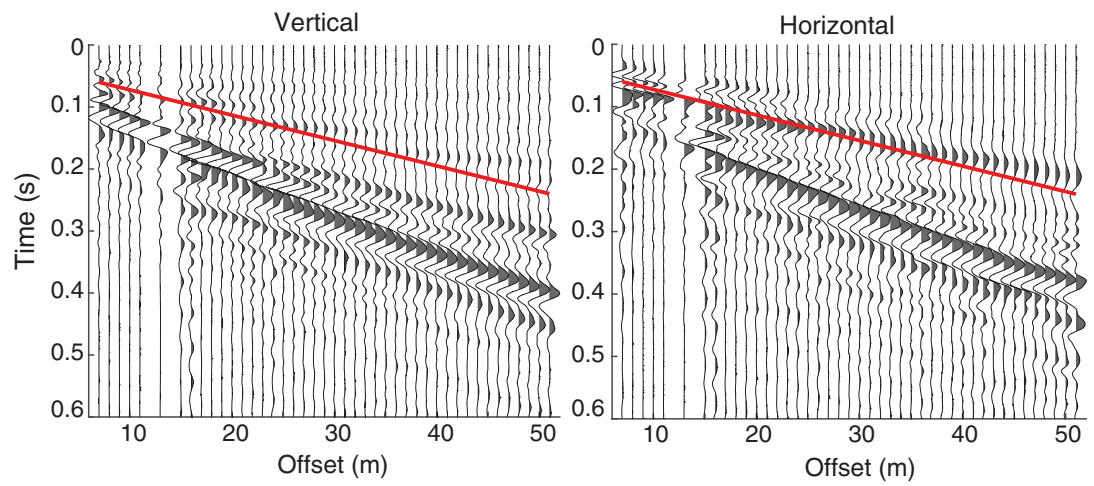

Figure 6. (a) Vertical and (b) horizontal component field shot gathers. The red line denotes location where top mute is applied to suppress first-arrival body waves. ity mute approach provides an opportunity to isolate the retrograde or prograde Rayleigh-wave modes prior to dispersion analyses. This approach can isolate the fundamental or higher mode arrivals that improve dispersion curve estimation capabilities and will in turn lead to improved S-wave velocity estimates with depth.

It is important to keep in mind that the technique demonstrated here separates wave modes based on the dominant particle motion. This technique does not distinguish between higher mode and fundamental mode. Therefore, if a given mode propagates in retrograde motion, it will appear in the prograde-muted dispersion image. If a given mode propagates in prograde motion, it will appear in the retrograde-muted dispersion image. As with any geophysical data analysis, caution should be exercised when identifying the modes in dispersion images. Although we point out that in the range of frequencies, we investigate here $(3-60 \mathrm{~Hz})$ and assume that higher modes propagate in prograde motion and the fundamental mode propagates in retrograde motion. It is worth highlighting a few studies that look at contradictions to this assumption. First, prograde motion of the fundamental mode has been modeled (Mooney and Bolt, 1966; Tanimoto and Rivera, 2005; Malischewsky et al., 2008); however, these studies observed prograde motion at frequencies much lower than our investigation $(0.1-0.3 \mathrm{~Hz})$. Second, De Nil (2005) reports that higher modes can transition from prograde motion to retrograde motion at the surface at high frequencies, typically a)

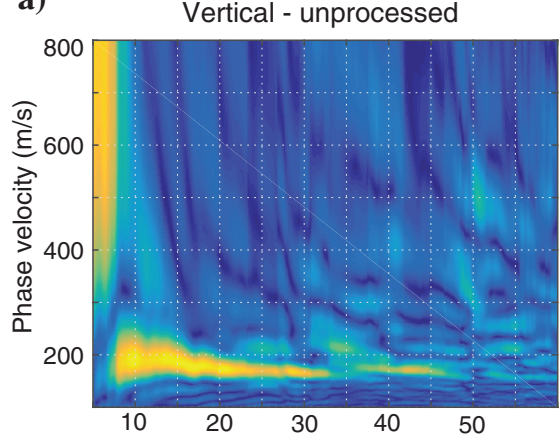

b)

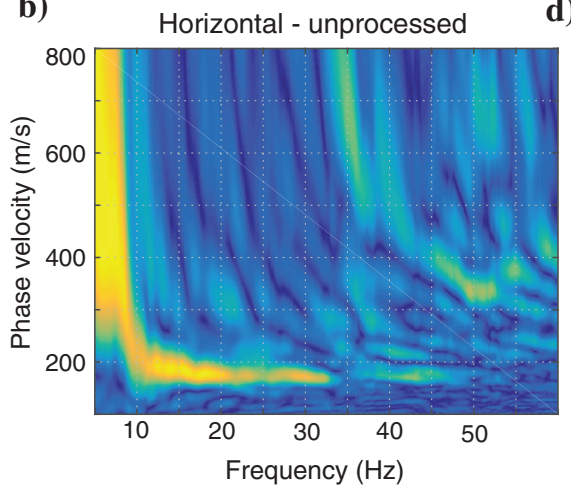

c)

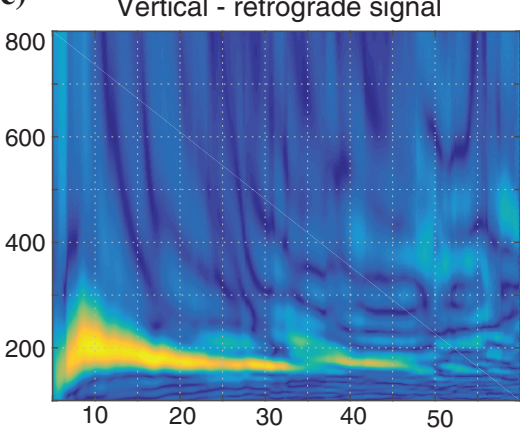

d)

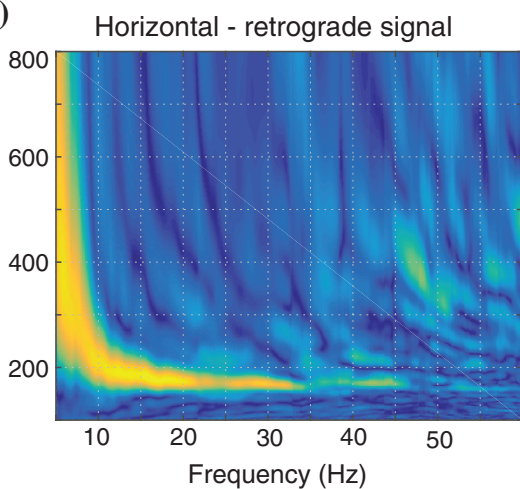

e)

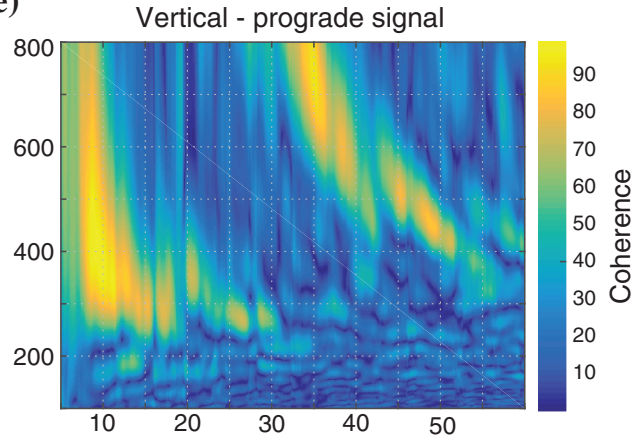

f)

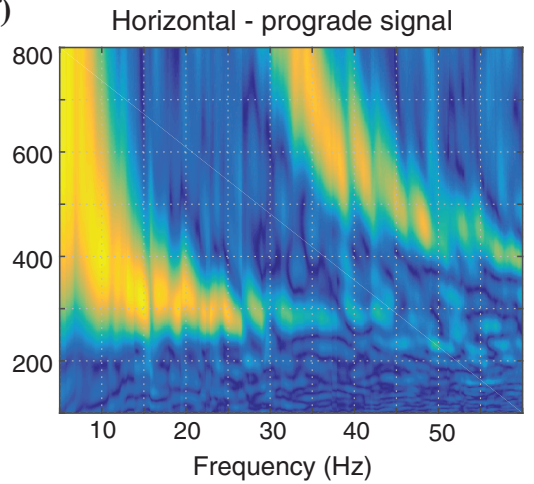

Figure 7. Phase velocity versus frequency coherence images for the field data with yellow denoting high coherence and dark blue denoting low coherence. (a) Raw vertical component data, (b) raw horizontal component, (c) vertical component after prograde polarity mute, (d) horizontal component after prograde polarity mute, (e) vertical component after retrograde polarity mute, and (f) horizontal component after retrograde polarity mute. Color scale indicates the coherence relative to maximum coherence in each plot. 
greater than $50 \mathrm{~Hz}$. This of course varies depending on the geologic model and the subsurface velocity distribution.

Aside from the possibilities of (1) energy from a single mode being split among the different dispersion images during muting or (2) mode misidentification, the other critical point to keep in mind relates to the low frequencies and the maximum receiver offset. The gradient velocity model highlights a potential shortcoming of this time-domain polarity mute. When retrograde and prograde signals overlap in time, the mute is applied based on whichever mode dominates. This means that energy can be mapped into the incorrect dispersion image. This will occur more often at lower frequencies, and one possible way to remedy this is to record greater offset so that the modes may separate in time. Alternatively, the imprint of these phenomena can be observed as abrupt lateral changes in dispersion image coherence or the abrupt appearance of energy as shown in Figure 4f. Taking these abrupt changes into account enables a quality check during dispersion curve picking. In future work, we plan to use time-frequency analysis to implement a frequency-domain version of this polarity mute and eliminate this problem.

\section{CONCLUSION}

We use the unwrapped phase information from combined vertical and horizontal inline component seismic recordings. From this phase information, we determine the instantaneous particle motion of the Rayleigh-wave arrivals. We then selectively mute arrivals that propagate in either retrograde or prograde motions. This polarity mute enables us to isolate the fundamental and higher order modes to more clearly define surface wave dispersion relationships in dispersion images. The improved coherency in dispersion images will in turn lead to more accurate $\mathrm{S}$-wave velocity estimates with depth in simple and complex geologic environments. We have shown two end-member environments and applied this new approach to field data. In the field data example, this approach allowed us to isolate higher modes and more confidently identify fundamental and higher mode dispersion curves.

\section{REFERENCES}

Aki, K., and P. G. Richards, 1980, Quantitative seismology: Theory and methods: W.H. Freeman.

Casto, D. W., C. Calderon-Macias, B. Luke, and R. Kaufmann, 2010, Improving MASW results for a site with shallow bedrock through the use of higher-mode data: GeoFlorida 2010, 1360-1368.
Casto, D. W., B. Luke, C. Calderon-Macias, and R. Kaufmann, 2009 Interpreting surface-wave data for a site with shallow bedrock: Journal of Environmental \& Engineering Geophysics, 14, 115-127, doi: 10 .2113/JEEG14.3.115.

Dal Moro, G., R. M. M. Moura, and S. S. Moustafa, 2015, Multi-component joint analysis of surface waves: Journal of Applied Geophysics, 119, 128 138, doi: 10.1016/j.jappgeo.2015.05.014.

De Nil, D., 2005, Characteristics of surface waves in media with significant vertical variations in elasto-dynamic properties: Journal of Environmental and Engineering Geophysics, 10, 263-274, doi: 10.2113/ JEEG10.3.263.

Dormán, J., and M. Ewing, 1962, Numerical inversion of seismic surface wave dispersión data and crust-mantle structure in the New York-Pennsylvania area: Journal of Geophysical Research, 67, 5227-5241, doi: 10 $.1029 / \mathrm{JZ} 067 \mathrm{i013p} 05227$.

Garland, G. D., 1979, Introduction to geophysics: Mantle, core and crust: W. B. Saunders Company.

Ivanov, J., R. D. Miller, S. Peterie, C. Zeng, J. Xia, and T. Schwenk, 2011, Multi-channel analysis of surface waves (MASW) of models with high shear-wave velocity contrast: 81 st Annual International Meeting, SEG, Expanded Abstracts, 1384-1390.

Jones, G. H. S., G. T. Maureau, and S. A. Cyganik, 1963, Air-blast coupling to prograde and retrograde surface waves: Journal of Geophysical Research, 68, 4979-4987, doi: 10.1029/JZ068i017p04979.

Liberty, L. M., and G. M. Gribler, 2014, Shear wave seismic velocity profiling and depth to water table - Earthquake site response measurements for Valley County, Idaho: Technical Report August.

Luo, Y., J. Xia, R. D. Miller, Y. Xu, J. Liu, and Q. Liu, 2009, Rayleigh-wave dispersive energy imaging using a high-resolution linear radon transform Geophysical Journal International, 179, 254-264, doi: 10.1111/j.1365246X.2009.04277.x.

Malischewsky, R. G., F. Scherbaum, C. Lomnitz, T. T. Tuan, F. Wuttke, and G. Shamir, 2008, The domain of existence of prograde Rayleigh-wave particle motion for simple models: Wave Motion, 45, 556-564, doi: 10.1016/j.wavemoti.2007.11.004.

Michaels, R., and R. B. Smith, 1997, Surface wave inversion by neural networks (radial basis functions) for engineering applications: The Journal of Environmental and Engineering Geophysics, 2, 65-76, doi: 10.4133/ JEEG2.1.65.

Mooney, H. M., and B. A. Bolt, 1966, Dispersive characteristics of the first three Rayleigh modes for a single surface layer: Bulletin of Seismological Society of America, 56, 43-67.

Park, C. B., R. D. Miller, and J. Xia, 1999, Multichannel analysis of surface waves: Geophysics, 64, 800-808, doi: 10.1190/1.1444590.

Rayleigh, L., 1885, On waves propagated along the plane surface of an elastic solid: Proceedings of the London Mathematical Society, si-17. 4-11.

Stokoe, K. H., and S. Nazarian, 1983, Effectiveness of ground improvement from spectral analysis of surface waves: Proceedings of the European Conference on Soil Mechanics and Foundation Engineering 1, 91-94.

Tanimoto, T., and L. Rivera, 2005, Prograde Rayleigh wave particle motion: Geophysical Journal International, 162, 399-405, doi: 10.1111/j .1365-246X.2005.02481.x.

Xia, J., R. D. Miller, C. B. Park, and G. Tian, 2003, Inversion of high frequency surface waves with fundamental and higher modes: Journal of Applied Geophysics, 52, 45-57, doi: 10.1016/S0926-9851(02)00239-2.

Zhang, S., 2011, Effective dispersion curve and pseudo multimode dispersion curves for Rayleigh wave: Journal of Earth Science, 22, 226-230, doi: $10.1007 / \mathrm{s} 12583-011-0175-8$. 